\title{
TENDENCIAS Y SITUACION ACTUAL DE LAS BIBLIOTECAS Y UNIDADES DE INFORMACIÓN EN AMÉRICA LATINA
}

\section{Gloria Ponjuán Dante}

\section{Título}

Tendências e situação atual das Bibliotecas e Unidades de Informação na América Latina

\section{Resumo}

Síntese do panorama geral das bibliotecas nacionais, públicas, escolares, universitárias e unidades de informação nos 18 países componentes da América Latina. Analisa o contexto profissional dos bibliotecários atuantes nessas instituições, bem como os elementos integrantes do circuito da informação eletrônica: edição, copyright, conservação. Conclui que o profissional da informação tem que aplicar métodos e ferramentas de gestão para uma atuação eficaz.

\section{Palavras-Chave}

Bibliotecas - América Latina; Profissional da informação - América Latina,

\section{INTRODUCCIÓN}

Intentar presentar un panorama resumido de las bibliotecas en nuestra Región y trazar sus tendencias no resulta sencillo; la generalización siempre conlleva al riesgo de no representar adecuadamente un panorama exacto del microentorno turbulento en que nos encontramos.

Aún así intentaré presentar en esta síntesis, un análisis de algunas variables y constantes que nos permitan presentar su evolución a las puertas de un nuevo milenio. Los datos y elementos aquí presentados han sido tomados de la bibliografía disponible, complementados con la percepción de la autora en diversos contactos con especialistas latinoamericanos y visitas a varios países de la Región.

CONTEXTO GEOGRÁFICO E HISTÓRICO 
ses (17 de habla hispana y uno de habla portuguesa, ver tabla 1).

Tabla 1

PAÍSES DE AMÉRICA LATINA
Argentina
Bolivia
Brasil
Chile
Colombia
Costa Rica
Cuba
Ecuador
El Salvador
Guatemala
Honduras
México
Nicaragua
Panamá
Paraguay
Perú
Uruguay
Venezuela

Estos países han sufrido a lo largo de su historia diferentes procesos de mezcla y síntesis a partir de las diferentes poblaciones y culturas indígenas, europeas y africanas. En este siglo puede también considerarse una nueva mezcla a partir de una creciente inmigración asiática hacia nuestros países.

Son estos mismos modelos e influencias de los que han permeado y determinado los paradigmas de desarrollo profesional imperantes en nuestra Región. En el contexto de dominación colonial (España, Portugal, Gran Bretaña, Francia u Holanda), en la etapa republicana adicionalmente por los Estados Unidos, y actualmente, la mezcla de múltiples influencias producto de los efectos de la globalización, donde prácticamente se pierden los límites de las nacionalidades y las etnías.

\section{CONTEXTO ECONÓMICO Y SOCIAL}

El desarrollo económico y social de nuestra Región está condicionado por algunos factores comunes, y por desigualdades importantes, a partir de los propios recursos naturales, la ubicación geográfica, los procesos de dominación colonial y determinadas coyunturas que no son objeto de nuestro trabajo. El Anuario Estadístico de UNESCO señala que nuestra Región muestra un $13 \%$ de analfabetismo, índice que varía de país en país, conforme a las diferencias antes 
señaladas.

Diferencias y coincidencias en el contexto económico y social de los países condicionan, por una parte, el desarrollo heterogéneo observado en los servicios bibliotecarios y de información, y por otra, estimulan la búsqueda de modelos, enfoques y soluciones similares para sostener una identidad regional.

\section{CONTEXTO PROFESIONAL}

En este contexto se abordará principalmente lo relativo a la evolución de:

- bibliotecas nacionales

- bibliotecas públicas

- bibliotecas escolares

- bibliotecas universitarias

- bibliotecas especializadas

- otras unidades de información

\section{Bibliotecas Nacionales}

Las Bibliotecas Nacionales mantienen su rol de salvaguarda del patrimonio documental de nuestra Región. El depósito legal en algunos casos es formal pero no efectivo, aunque cuenta con la legislación nacional que lo ampara. Por este motivo es raro encontrar en bibliotecas del Primer Mundo, documentos que no forman parte del Patrimonio de las Bibliotecas Nacionales de la Región.

En la maioría de los casos, estas bibliotecas sostienen su expertizaje profesional en el ámbito nacional, principalmente en funciones normalizadoras, en la aplicación de formatos, etc. Se ha generalizado la utilización de tecnologías de información en las bibliotecas nacionales de casi todos los países latinoamericanos, en buena parte de ellos se cuenta con catálogos automatizados de colecciones, y en algunas, con catálogos en línea. Sin embargo, no se distinguen por aplicar principios gerenciales a su actividad, aspecto que pudiera contribuir a optimizar sus resultados y elevar su eficiencia.

Desde 1988, 18 países latinoamericanos y caribeños colegiaron sus esfuerzos en ABINIA (Asociación de Bibliotecas Nacionales de Iberoamérica). Esta Asociación ha trazado proyectos interesantes de cooperación como el Catálogo colectivo de fondos antiguos de los siglos XV-XIX y la Historia de las Bibliotecas Nacionales de Iberoamérica: pasado y presente uniéndose y participando en el proyecto de UNESCO acerca de la Memoria del Mundo.

En líneas generales, persisten los esfuerzos con relación a las bibliografías nacionales, aunque sólo pueda referirse a; 33\% de ellas actualizadas.

Algunos países han dedicado grandes inversiones al fortalecimiento de la infraestructura de sus Bibliotecas Nacionales, como el caso de Venezuela, y más recientemente Panamá que en un plazo muy corto, han logrado un salto sustantivo en su organización y servicios, destacándose la próxima conexión en línea de su catálogo con Bibliotecas filiales en otras provincias. Otros pocos, acometen proyectos de edición en CD-ROM.

\section{Bibliotecas públicas}

En muchos países latinoamericanos, la 19 
propia Biblioteca Nacional forma parte del sistema de bibliotecas públicas por el hecho de prestar servicios a la comunidad. El concepto de biblioteca pública se ve enriquecido en la Región por el de biblioteca rural, biblioteca popular y en algunos países, aún por el de bibliotecas escolares. También han surgido otras dedicadas a etnías locales.

Las bibliotecas públicas por lo general son municipales, aunque también pueden ser parroquiales, comunales o de cooperativas. En algunos países se han integrado en una red, muchas veces subordinadas al Ministerio de Educación, lo que potenció sus actividades por el beneficio de la interrelación mutua.

En muchas bibliotecas públicas se privilegía la atención a los niños y adolescentes y también se verifican nexos y coordinaciones con las escuelas. Se les brinda apoyo en actividades extraescolares, desarrollan actividades de promoción de la lectura, la Hora del Cuento, Teatro, el Club de Madres, etc. Este tipo de bibliotecas no siempre cuenta con personal profesional y debidamente capacitado para atender adecuadamente toda esta gama de actividades que constituyen un derecho ciudadano ineludible.

Existen otras que han asumido un cierto nivel de especialización para atender a ciegos y débiles visuales.

Los servicios de extensión bibliotecaria, las bibliotecas circulantes, las bibliotecas móviles, las exposiciones de hechos históricos, culturales, etc., también forman parte de sus principales características.
No predomina en este ámbito el uso de las nuevas tecnologías ni las técnicas y herramientas gerenciales. Muchas bibliotecas públicas de la Región se mantienen dentro de los sistemas tradicionales, esto no ha impedido ni ha inhibido el desarrollo de inciativas de gran creatividad y de una voluntad marcada de atender al usuario con dedicación y entrega, lo que podría ser potenciado con la aplicación de técnicas de gestión y la introducción de tecnologías. Un ejemplo de ello, es notable el impacto del proyecto del Biblio-Metro desarrollado en Santiago de Chile en la Dirección de Bibliotecas, Archivos y Museos (DIBAM).

Existen bibliotecas públicas asociadas a entidades privadas, por ejemplo, las Cajas de Compensación en Colombia, donde se realizan múltiples servicios e iniciativas hacia la comunidad en general y en particular hacia la población infantil, con préstamos especiales a los hijos de los asociados, con actividades de promoción de la lectura.

Aún así, las bibliotecas públicas por lo general enfrentan un conjunto de dificultades. A modo de ejemplo, los principales problemas identificados en las bibliotecas públicas de Colombia han sido reportados en el trabajo de dos especialistas de ese país $\left({ }^{1}\right)$. Entre ellos incluyen: recursos humanos insuficientes y poco preparados; falta de presupuestos propios; escasez de recursos para adquisiciones; coleciones que no responden a las necesidades reales de la comunidad; ausencia de marco jurídico que

${ }^{1}$ ALVAREZ, M. y M. Mejía. Colombia. In: MORALES, E., comp. Bibliotecología Latinoamericana: un panorama general. México: CUIB, 1989. p.25-47.

Inf.Inf., Londrina, v. 4, n. 1, p. 17-35, jan./jun. 1999 
reconozca a los sistemas de bibliotecas; problemas político-administrativos; carencia de políticas; problemas de carácter técnico y de comunicación; deficiencias de sus servicios. Este panorama con pocas diferencias, es el mismo que se observa en la mayoría de los países de la Región.

De la misma manera puede señalarse que no todos los países han desarrollado armoniosamente sus redes de bibliotecas públicas, por lo que aún se encuentran concentradas en grandes zonas urbanas. Las zonas apartadas, las poblaciones indígenas o las comunidades en condiciones especiales no siempre cuentan con bibliotecas de este tipo.

UNESCO apoyó en América Latina el proyecto piloto de las bibliotecas públicas. Así se creó la Biblioteca Pública Piloto de Medellin (1954) que al concluir el proyecto y pasar a ser administrada localmente, muestra una interesante experiencia de cómo transitar de un financiamiento internacional a las condiciones de nuestros países, intentando gerenciar su supervivencia sin perder lo esencial de sus servicios y logros. Esta biblioteca es un buen modelo en lo que a la aplicación de métodos gerenciales se refiere, lo que la ha llevado a obtener en encuestas realizadas a sus usuarios, índices de satisfacción con los servicios equivalentes a un $100 \%\left(^{2}\right)$.

\section{Bibliotecas escolares}

Las bibliotecas escolares constituyen el

2 PALOMINO, G. I. Comunicación personal. 1996. eslabón más importante y lamentablemente el más débil de la cadena informacional. En ellas recibirán sus primeros estímulos los futuros usuarios de la información. Su desarrollo guarda estrecha correspondencia con las prioridades que cada gobierno le brinde al sistema de educación pues sus presupuestos generalmente dependen de ello. En muchos casos las salas infantiles y juveniles de las bibliotecas públicas suplen o complementan las ausencias o limitaciones de las bibliotecas escolares. En algunos países se ha suplido la falta de bibliotecarios escolares con la capacitación de maestros-bibliotecarios, un ejemplo de ello, es el programa que se implementó con buenos resultados en Argentina.

En este rubro también se observa la tendencia a crear los llamados Centros de Recursos de Aprendizaje, organismos que en esencia mantienen los mismos objetivos que las bibliotecas escolares, pero constituyen un modelo más flexible e integral, no sólo por la diversidad de los recursos tecnológicos y de información que incorporan a sus servicios y ofrecen a sus alumnos, sino por la concepción más acorde a la metodología moderna del proceso de enseñanzaaprendizaje, impulsado por las reformas educacionales emprendidas en diversos países de la Región.

Algunos vicios siguen presentes en este nivel. Los niños siguen sufriendo como castigo, el ir a la Biblioteca. Todavía existen profesores que no distinguen los beneficios que les aporta a su propio desarrollo profesional y como consecuencia no estimulan el uso de la información como apoyo fundamental al proceso 
educativo de sus alumnos. Muchas de estas unidades, carecen de la visión estratégica de introducir a sus usuarios en el mundo fantástico de la consulta de los libros y del acceso a la información y por lo mismo, se emplean con múltiples propósitos no siempre vinculados a sus fines.

En este ámbito es bastante frecuente encontrar marcadas diferencias entre las bibliotecas escolares del sector público y privado, ello naturalmente obedece a factores de financiamiento. Sin embargo y pese a la importancia de ese factor, muchas consiguen resultados muy alentadores cuando los directivos del establecimiento educacional le dan debida prioridad a este aspecto.

En algunos países y un tanto excepcionalmente pero cada vez con mayor énfasis, se han organizado actividades para orientar a los niños en el uso de Internet, elemento que puede potenciar extraordinariamente su proyección futura hacia estos recursos de información.

El índice de bibliotecas escolares es muy bajo en determinados países, existiendo mayoritariamente las llamadas bibliotecas de aula que en realidad son colecciones de literatura para niños ubicadas en diferentes salones de clase y manejadas por el maestro. En 1989, Paraguay reportaba por ejemplo, la existencia de 54 bibliotecas escolares, con 3 bibliotecarios profesionales $\left({ }^{3}\right)$.

\footnotetext{
${ }^{3}$ MORA, D. V . Paraguay. In: MORALES, E., (comp.) Bibliotecología Latinoamericana: un panorama general. México : CUIB, 1989. p.107-117.
}

\section{Bibliotecas universitarias}

Las bibliotecas universitarias son, dentro de la tipología de unidades de información mencionadas, una de las que al nivel de toda la Región mantiene con relativa uniformidad un grado de atención, y recursos que superan los de otras bibliotecas. Generalmente se organizan en una red o sistema, de bibliotecas de facultades y departamentos y una biblioteca central. Por lo general, constituyen un gran apoyo para los programas docentes, y brindan servicios especializados a los investigadores y profesores de las mismas.

La infraestructura de las bibliotecas universitarias es por lo general, una de las más fuertes, contando en la mayoría de los casos con personal calificado, presupuestos medianamente adecuados para el desarrollo de colecciones, recursos computacionales, medios de reproducción y comunicación.

En algunos países se han dado muestras notables de la gestión de recursos informacionales de las universidades mediante el empleo de las nuevas tecnologías de información y comunicación. Es en este ámbito donde se da con mayor frecuencia la búsqueda e implementación de sistemas integrales para la automatización de sus procesos y servicios. Son también las que muestran mayores progresos en cuanto a los mecanismos de acceso a la información y bases de datos en línea o local (a través de CD-ROM) de una variada gama de disciplinas y especialidades. Muchas generan sus propias publicaciones impresas o electrónicas y sostienen un intercambio regular con instituciones afines en la Región. 
En México, el Colegio de México cuenta con una ejemplar biblioteca automatizada y con una valiosa colección especializada en Ciencias Sociales.

En Chile, los esfuerzos y progresos del sistema de bibliotecas universitario más tradicional, incluyendo algunos casos del sector de educación privado, son muy alentadores. Se destacan entre ellos: el sistema de bibliotecas de la Unviersidad de Chile, de la Pontificia Universidad Católica, de la Universidad Austral, de la Universidad Católica de Valparaíso, de la Universidad Técnica Federico Santa María y también de otras como la Universidad Diego Portales entre las universidades del sector privado.

También pueden señalarse los de la Universidad Católica de Perú, y la Pontificia Universidad Javeriana de Bogotá, Colombia. Otro ejemplo particularmente importante ha sido el de la Universidad de Colima, México que se ha convertido en una naciente industria de creación de discos compactos, cuyos servicios se brindan mediante una biblioteca electrónica que actualmente se irradía, mediante un proyecto de extensión, hacia toda la comunidad.

En algunas universidades, existen relaciones locales con empresas e instituciones investigativas y productivas. Las relaciones "universidad-empresa" constituyen muchas veces un elemento de interacción bastante eficaz entre estos dos sectores. En tal sentido las bibliotecas universitarias actúan como centros de información especializada, desarrollando servicios informativos empleando las últimas tecnologías disponibles, aplicando nuevos mo- delos de gestión y liderando los procesos de cambio.

\section{Bibliotecas especializadas}

Las bibliotecas especializadas se han distinguido en nuestra Región al exhibir interesantes experiencias de cooperación y mostrar una mayor adaptación a los cambios que inevitablemente se operan en nuestro sector informacional.

En muchos casos han transitado del trabajo con documentos, al trabajo con información, evolucionando hacia Centros de Información. Se han desarrollado importantes experiencias de cooperación conducentes a contar con sus propios productos y servicios con valor agregado. Es el caso del sector agrícola, donde el sistema AGRINTER, creado y liderado por el CIDIA (Costa Rica) estableció y desarrolló un esfuerzo regional que se vinculó al Sistema Internacional AGRIS de la FAO. El sector de las ciencias biomédicas también ha sido un paradigma en la Región a partir de los trabajos de la Biblioteca Regional de Medicina (BIREME, Brasil), actualmente Centro de Información para las Ciencias Médicas de América Latina y el Caribe, productor de la Base de Datos LILACS, equivalente al del Index Medicus de América Latina, que a su vez ha apoyado otros proyectos latinoamericanos como el CD-ROM de Red de Redes y otros esfuerzos en el campo de las publicaciones electrónicas.

El sector del petróleo tiene fuertes pilares en INTEVEP de Venezuela, PEMEX de México o PETROBRAS de Brasil. El sector industrial también muestra un buen desarrollo de sus 
servicios de información en muchos países, como México, Brasil, Costa Rica, y otros.

Las instituciones dedicadas a la investigación científica de diversos países han desarrollado en buena medida sus unidades de información especializada. Así la Biblioteca Marcel Roche del IVIC de Venezuela, ha sido reconocida por parte de UNESCO como Centro para el suministro de documentos especializados para nuestra Región. En México el 60\% de la investigación es desarrollada por la Universidad Nacional Autónoma de México por lo que también es reconocida su biblioteca y otros centros especializados como un ejemplo de apoyo a la investigación científica y humanística.

\section{Otras unidades de información}

Algunos países de la Región han desarrollado los llamados Institutos de Información. En 1954, Brasil creó el Instituto Brasileiro de Informação em Ciência e Tecnologia (IBICT) para cumplir con determinados objetivos: organización del catálogo colectivo nacional, compilación de bibliografías especializadas y mantenimiento de una biblioteca especializada. Desde entonces, su actividad ha evolucionado y ha jugado un notable rol en la creación de centros y servicios de información especializados, en la creación de diversos proyectos y programas con fines diversos (CONMUT, ANTARES) y en la formación de programas de postgrado en Ciencia de la Información en coordinación con la UFRJ, entre otros.

En Cuba, en 1963 se creó el Instituto de Documentación e Información Científica y Téc- nica (IDICT) con el objetivo de desarrollar el Sistema Nacional de Información en Ciencia y Tecnología. En sus 35 años, el IDICT ha sido pionero de la introducción, de nuevas tecnologías de información a nivel nacional. Así realizó un servicio de teleacceso a bases de datos en otros países, produjo el primer disco compacto cubano, estableció la primera red de intercambio automatizado de información con servicios de correo electrónico, gopher, boletín electrónico, etc. Ha impulsado el desarrollo profesional, la creación de multimedia, los servicios de consultoría, las investigaciones y la generación de una política nacional de información.

En algunos países han comenzado a surgir algunos servicios especializados de consultoría, generalmente dentro del sector privado. Van surgiendo corredores de información independientes, $\mathrm{u}$ organizados en entidades dedicadas a brindar este servicio a clientes o sectores puntuales.

Los archivos administrativos o registros administrativos muestran un bajo nivel de desarrollo en nuestra Región. Generalmente predominan los archivos de tipo histórico, aunque no siempre cuentan con recursos que les permita proteger y conservar los documentos que atesoran.

Es incalculable el beneficio que pueden alcanzar las organizaciones si controlan adecuadamente sus registros administrativos y logran integrar todas las actividades de información de su organización mediante una gerencia de recursos de información dentro de los máximos niveles de dirección de la entidad. 
Los centros de investigación especializados en Ciencias de la Información constituyen a su vez, otra de las unidades de información que han mostrado una mayor presencia en nuestra Región en los últimos años. Argentina, México, Cuba, Colombia, Brasil, cuentan con unidades de investigación de diferente magnitud. Cabe destacar la trascendencia y el impacto de los proyectos y productos implementados y liderados por CLADES (CEPAL) a nivel de la Región, en especial lo relativo a la generalización de resultados de estudios realizados por sus especialistas en la línea de gestión de información.

En algunos países se han gestado organizaciones encargadas de ofrecer determinados servicios a partir de las nuevas tecnologías como la preparación de páginas WEB, productos multimedia para diversos fines, publicaciones electrónicas, servicios de conectividad, y otros.

También se gestan organizaciones que desarrollan cursos, mantienen la distribución de publicaciones, softwares especializados para bibliotecas, editan textos, prestan servicios de reproducción a los bibliotecarios y otros profesionales de la información, etc. Ejemplo de estos, podemos encontrar en Argentina, México, Chile, y otros países.

\section{Otros elementos del contexto profesional}

Dentro del contexto profesional existen esfuerzos dignos de ser considerados en este panorama.

La formación de profesionales ha mostrado avances notables, principalmente en los últi- mos años. La revisión de los programas de estudio o pensium de las carreras de bibliotecología se ha convertido en la preocupación permanente en múltiples países como Argentina, Brasil, Colombia, Cuba, Chile, y otros. Los estudios de postgrado, se han intensificado habiéndose creado diversas posibilidades de estudios de Maestría y doctorados, aspecto que hasta hace pocos años era exclusivo de Brasil. En muchas de las Universidades lationamericanas que han acometido este esfuerzo se ha contado con la cooperación de diversas universidades españolas. Brasil por su parte también ha incrementado los programas de maestría y doctorados.

Especial mención debe hacerse al esfuerzo de los países de Mercosur con vistas al fortalecimiento de sus programas de estudio y otras acciones de interés común, que llevó a la constitución de la Asociación de Escuelas de Bibliotecología del Cone Sur (ASEBICS).

Los estudios de educación continua constituyen una práctica regular para los profesionales latinoamericanos destacándose los esfuerzos tanto de las Asociaciones Profesionales, como de las Escuelas de Bibliotecología y otras instituciones, principalmente públicas, organizadas en Argentina, México, Chile, Cuba, Brasil, Costa Rica y Colombia.

Los eventos de intercambio profesional se han sistematizado en casi todos los países de la Región. Resulta estimulante ver como Argentina, Brasil, Chile, Colombia, Venezuela, Uruguay, Costa Rica, México y Cuba, desarrollan con cierta regularidad sus eventos. Nuestra Región también 
ha sido sede de eventos de IFLA y FID.

Las Asociaciones profesionales han retomado otros esfuerzos en favor de la profesión. Puede mencionarse también su esfuerzo en función de las ediciones de la especialidad, principalmente las publicaciones seriadas, editadas también por instituciones dedicadas a la investigación y al desarrollo profesional. Así, nuestra Región muestra importantes resultados en la publicación de revistas especializadas, boletines, monografías y otros textos. Se han preparado diversos productos en CD-ROM (por ej. Bases de datos latinoamericanas, Internet para bibliotecarios) auspiciados por la UNESCO, y se han generado esfuerzos cooperativos como la BD INFOBILA y otros. También la Región cuenta con Asociaciones Regionales, como AIBDA, ABIPALC, ACURIL, RIALIDE, SPOTCOM y representaciones de las asociaciones internacionales como FID-CLA e IFLA-LAC. La UNESCO ha organizado otros esfuerzos importantes como el Sistema de Bibliotecas Asociadas.

La investigación, elemento ausente mayoritariamente en nuestro Sector ha dado muestras de fortalecimiento, como se menciona en otra parte de este documento. Brasil, sin duda, encabeza este esfuerzo con un sólido programa de investigación apoyado por el CNPq y con la participación de las principales Universidades y de la ANCIB (Associação Nacional de Pesquisa em Ciência da Informação). Esta última tuvo la iniciativa de organizar el pasado año en Rio de Janeiro en el marco de su Tercer Encuentro Nacional de Investigación en Ciencia de la Información, el Primer Foro Latinoamericano de
Investigación en Ciencia de la Información con la participación de representantes de Brasil, Cuba, México, Perú y Uruguay.

Desde hace cuatro años, se han retomado los Encuentros de Educadores e Investigadores en Bibliotecología, Archivología y Ciencias de la Información de Iberoamérica y el Caribe, que abordan diferentes temas relativos al pregrado, postgrado, investigación y educación continua. Como acuerdo de estas reuniones se ha constituído la Asociación de Educación e Investigación en Bibliotecología, Archivología, Ciencia de la Información y Documentación de Iberoamérica y el Caribe, como continuadora de ALEBCI, creada hace varios años en Panamá.

Otro esfuerzo notable en la comunicación, los servicios y el intercambio profesional se logra mediante las diversas listas de discusión existentes. México, Chile, Cuba, Argentina, Brasil cuentan con experiencias de esta naturaleza y participación en otras de carácter regional para grupos de interés común (educadores, por ej.).

\section{BALANCE DE LA SITUACIÓN ACTUAL}

América Latina ha sostenido sus servicios bibliotecarios y de información en medio de la turbulencia y crisis económica general que ha vivido la Región. Paulatinamente las nuevas tecnologías de información han ido incorporándose a procesos y servicios bibliotecarios. Internet hizo su entrada en el sector y se aprecia el empleo de este recurso principalmente para ofrecer servicios a los usuarios, en un plano más personalizado. Las bibliotecas latinoamericanas tienen una baja 
representatividad en las páginas WEB de Internet. Esto hace pensar que los servicios de información y bibliotecas latinoamericanos, son más consumidores que productores, de la información que se encuentra en Internet.

Se aprecian importantes esfuerzos para utilizar nuevos portadores en la difusión de acervos, y en particular, colecciones de publicaciones seriadas. Esto permite ofrecer acceso a las bases de datos latinoamericanas, y un aporte a la conservación de acervos.

Aún cuando persisten los trabajos cooperativos de algunas grandes redes de información, la falta de un financiamiento sostenido y nuestras debilidades para generar productos y servicios realizables, ha debilitado considerablemente estos proyectos. El mayor crecimiento se observa en unidades de información que pertenecen a unidades de empresas y organizaciones individuales. Los proyectos cooperativos se han sostenido, aunque modificando sus objetivos. La tendencia a crear y mantener bases de datos cooperativas ha dado paso a esfuerzos por favorecer la accesibilidad y se enfocan colectivamente los problemas del momento.

En esta última década, también se evidencia un incremento en acciones que favorecen el desarrollo profesional de los recursos humanos de las unidades de información. Objetivamente, el cambio y el mejoramiento, en época de escasos recursos, debe realizarse a partir de la potenciación del recurso más valioso: el humano.

Valentim resume la adopción de nuevos paradigmas en las bibliotecas en la siguiente tabla:

\begin{tabular}{|l|l|}
\hline BIBLIOTECA - MODELO ANTIGUO & BIBLIOTECA - MODELO NUEVO \\
\hline Estructura jerárquica & Estructura horizontal \\
\hline Enfoque de acervo & Enfoque en la información \\
\hline Organización estática & Organización dinámica \\
\hline Trabajo desarrollado en servicios & Trabajo desarrollado en proyectos \\
\hline Gerencia centralizada & Autogerencia \\
\hline Conocimientos específicos & Conocimientos amplios \\
\hline Relaciones competitivas & Relaciones cooperativas \\
\hline Motivación individual & Motivación de equipos \\
\hline Acciones controladas & Acciones innovadoras \\
\hline Atención personal & Atención remota \\
\hline Investigación en el sitio (in situ) & Investigación remota \\
\hline Acervo lineal & Acervo óptico \\
\hline Lenguaje controlado & Lenguaje libre \\
\hline Entrada de datos, referencias & Entrada de texto completo \\
\hline Servicios in situ & Servicios on line \\
\hline Productos impresos & Productos electrónicos \\
\hline Uso de sistemas aislados & Uso de sistemas integrados \\
\hline Uso de medios únicos & Uso de multimedia \\
\hline
\end{tabular}


En época de escasez de recursos, lograr efectividad, productividad, impacto... hacer más con menos y obtener resultados con esfuerzos concentrados, principios que conocemos por Bradford, Pareto y otros, constituye no una mera estrategia sino una impostergable necesidad para sobrevivir.

En las variables seleccionadas por Valentim, se aprecía una transición gradual, que quizás no ha sido tan rápida como se hubiera deseado. Se aprecía también, una notable influencia de las tecnologías de información y la importancia de las técnicas y herramientas gerenciales en el cambio de paradigmas.

Estos elementos muestran diferencias importantes en la evolución de las bibliotecas y unidades de información, en correspondencia con los niveles de desarrollo económico y social, así como de las infraestructuras que cada país ha logrado alcanzar.

RETOS Y PANORAMA DEL NUEVO MILENIO

El nuevo milenio que se avecina, será abordado por las bibliotecas y unidades de información de América Latina en medio de un conjunto de retos que debe enfrentar el sector de información. Se intentará hacer una síntesis de éstos.

\section{- la sociedad de la información}

Si se cumple lo que conceptualmente se define como una sociedad de la información, deberían estar presentes tres características: que se utilice la información como recurso económico en las organizaciones; que se aprecíe un mayor uso de información en la comunidad; que se desarrolle un sector de información dentro de la economía de cada país.

Indudablemente, estas tres características están presentes en nuestra Región, sólo que el desarrollo desigual de las economías, de sus componentes y los efectos de la política económica y la globalización, pueden hacer el proceso más lento o más rápido en función de determinados intereses y prioridades locales.

\section{- las autopistas de información}

Las autopistas de información constituyen la convergencia de las tecnologías de computación y de comunicación, aspecto que inevitablemente será de utilización masiva en el futuro.

La generalización o uso extensivo por diferentes niveles de la sociedad guarda relación con los niveles de desarrollo educacional y social de los países, así como, con el propio desarrollo de la infraestructura y nivel de vida de las poblaciones. El analfabetismo, la escasez de recursos, la pobreza, no son compatibles con las autopistas de información. Tampoco lo es, la baja cultura informacional que tiene la población en general, y lo que es más grave, la propia población profesional.

El uso generalizado de las autopistas también requerirá que la oferta de contenidos que transitan por la misma, responda a los intereses de los usuarios. La información deberá ser públi- 
ca, y fluir en nuestra lenguas y dialectos. La existencia de una autopista que sólo transmita en inglés y que responda principalmente a las transacciones comerciales, la publicidad, y otros intereses particulares, y la existencia de autopistas privatizadas donde haya que pagar peaje para entrar en cualquier sitio, no favorece el desarrollo de los países en desarrollo, que son mayoritarios, por no decir todos, los de América Latina.

\section{- la conservación}

El desarrollo de las tecnologías de información ha impuesto nuevos retos.

Los pergaminos se conservan desde hace 1.200 años y es de esperar que tengan un ciclo de vida aún mayor; los documentos en papel se conservan desde hace más de 700 años y es de esperar que con los estudios que se hacen para desarrollar un papel permanente, garantice su vida por más de un miliar de años. Tanto uno como otro soporte son perfectamente legibles. La película fotográfica se emplea hace 150 años y sus expectativas de vida están en el orden entre 300 y 1000 años.

El resto de los soportes aún constituyen una gran incógnita. En el Informe Mundial de Información 1997/98 publicado por UNESCO se plantea que la vida media de las cintas magnéticas empleadas desde hace 50 años, es de 30 años, de los disquetes 30 años y de los CDs entre 30 y 50 años, sin que pueda asegurarse aún, su compatibilidad con otras tecnologías que vayan surgiendo en el futuro.

Evidentemente, alguna solución surgirá para estos soportes. Al igual que las computadoras ahora enfrentan el llamado "Efecto 2000", pueden presentarse en el futuro, otros y determinados "Efectos" para la reutilización o conversión de registros electrónicos mediante nuevas tecnologías que surgirán en el futuro.

Al margen de estos problemas que se derivan del desarrollo tecnológico, aún los países latinoamericanos deberán seguir enfrentando los retos de intentar recuperar importantes y valiosos fondos afectados por los efectos de la humedad, las plagas, las altas temperaturas, y las consecuencias de malos manejos de documentos.

\section{- la edición de libros}

Aún cuando es prácticamente imposible determinar los volúmenes de documentos que se editan a escala mundial. Se estima que circulan 9.000 periódicos y 50.000 publicaciones seriadas dedicadas a los aspectos científicos y académicos. Por la importancia de las ediciones para la vida científica, cultural y social de las naciones y por constituir éstas las principales fuentes que hoy forman parte de los acervos latinoamericanos y que éstas constituyen la fuente principal de muchas de las investigaciones que se realizan, el seguimiento de este mundo editorial constituye un reto permanente, para los profesionales de la información.

La tecnología también ha ejercido grandes influencias en los cambios acaecidos en las esfera editorial: los desarrollos en la computación y en la reprografía unidos a las oportunidades derivadas de las redes globales han generado un 
salto de grandes proporciones, reduciendo considerablemente los costes de producción, aunque esto definitivamente no se ha visto reflejado en los precios de venta. En la cadena de producción-distribución se obtienen los mayores beneficios. Estos avances y beneficios no alcanzan el nivel que experimentan en el Primer Mundo, en nuestra Región. Aún así, México y Argentina son los países latinoamericanos que muestran una industria editorial más desarrollada, no por eso logran competir con otras regiones, a pesar de ser fuertes exportadores y de dominar el mundo editorial de habla española. Brasil también tiene una industria editorial bastante desarrollada, lo que le permite autoabastecerse.

En el mundo editorial actual tres retos se identifican: el avance tecnológico que obliga inversiones constantes; los asuntos asociados al derecho de autor o copyright y el control de la producción editorial.

Muchos sistemas de bibliotecas apoyan sus procesos en los códigos de barra para la organización y control de sus colecciones. El sostener una sólida interacción entre editoriales y sistemas de bibliotecas, permitirá no afectar la organización y control logrados. Los sistemas de bibliotecas deberán ejercer alguna presión sobre los editoriales y distribuidores para alcanzar precios preferenciales que les permitan contar con una mayor representatividad de las obras en las mismas. Por otra parte, darle prioridad a la producción intelectual latinoamericana y lograr una política de precios que estimule la compra de obras latinoamericanas, puede contribuir a reforzar nuestra identidad y elevar la cultura de las nuevas generaciones.

\section{- el copyright y el copyleft}

Aún cuando los asuntos asociados a la propiedad intelectual todavía se encuentran bastante incipientes en todo el mundo y en América Latina aún más, el nuevo milenio enfrentará nuevas formas de generación y de transmisión de conocimientos. Esto obligará a buscar soluciones para lograr la protección de la creación de unos por otros que indiscriminadamente abusan de las potencialidades de la tecnología para disfrutar, sin autorización, de los frutos del esfuerzo de aquellos que los producen. A modo de ejemplo, la protección de productos multimedia puede ser representativa, ya que al integrar diversos medios, protegidos cada uno por regulaciones diferentes al nivel de cada país, obligan a preparar regulaciones especiales para estos productos integradores. Este elemento ético es algo que deberá ser objeto de especial atención principalmente en nuestra Región.

En alguna medida los órganos de alcance regional, deberán fomentar el establecimiento de políticas nacionales que regulen uniformemente dentro de América Latina, la reproducción sin autorización, de documentos en papel o formato electrónico, imágenes etc., o apoyen la promulgación de una regulación a escala mundial, que puede constituir la solución general para abordar las consecuencias de la globalización.

El empleo de algunos productos, como softwares, que puedan ser empleados y modificados libremente, seguirá siendo estimulado principalmente por organizaciones internacionales y

Inf.Inf., Londrina, v. 4, n. 1, p. 17-35, jan./jun. 1999 
asociaciones profesionales.

\section{- la gestión de recursos y la inteligencia organizacional}

Las organizaciones van valorando paulatinamente la importancia de la información. Mientras más competitividad existe, mayor importancia adquiere la información como un recurso crítico para la supervivencia y el éxito organizacional.

El posicionamiento de los bibliotecarios o profesionales de la información en la gestión de recursos de información de una organización, constituye un reto importante que debe ser asumido en forma intensiva en los países latinoamericanos. Para ello resulta determinante lograr intensificar la formación de postgrado y educación continua, derivando fuerzas hacia la preparación de gerentes de infomación para las organizaciones (CIOs). No por gusto planteó Baumard: "el desarrollo de las capacidades nacionales de inteligencia deben estar dirigidas hacia las habilidades necesarias para interpretar la información y hacer que tenga sentido".

Sólo mediante un posicionamiento estratégico de nuestros profesionales en estas funciones permitirán a las organizaciones sostener un monitoreo del medio y el desarrollo de la función de inteligencia organizacional, generando productos del proceso de inteligencia. Nuestros países se alejan cada vez más de los avances que se obtienen en el Primer Mundo, en este terreno. Si hay un camino importante que recorrer, a un paso acelerado, es éste. A medida que avance la globalización, los mayores perdedores, invariablemente seguirán siendo los países del Tercer mundo. Por tanto, el desarrollo de capacidades que permitan interpretar y comprender los ambientes económicos y sociales; generar una base de conocimientos compartida orientada hacia la definición de acciones concertadas para enfrentar los retos de la globalización e implementar estrategias que promuevan un desarrollo social y económico, deben ser prioridades a considerar.

\section{- la gestión de recursos humanos}

Producir un vuelco en la gestión de los recursos humanos, es otro de los grandes desafíos a enfrentar en este contexto. La introducción de nuevos estilos para la organización y tratamiento del recurso humano, el más valioso disponible en el sector de información y en todos los ámbitos en general, ha sido limitada. Las generaciones que surgen en medio de estas grandes transformaciones y cambios de paradigma, deben asumir nuevos estilos sin perder todo lo valioso y susceptible de conservar de los estilos vigentes. En las unidades de información y bibliotecas latinoamericanas, el cambio de la cultura organizacional; el desarrollo del trabajo en equipos; la organización siguiendo los procesos productivos; la gestión de la calidad total como filosofía donde impere un verdadero mejoramiento continuo y un empowerment (potentación) de las fuerzas productivas, constituyen líneas a considerar.

Grandes esfuerzos deben dedicarse a la potenciación de capacidades de liderazgo. Aún cuando nuestro sector ha dado pasos importantes en este sentido y se han ganado espacios a 
partir de un determinado nivel de liderazgo, el salto debe ser sustancialmente mayor para lograr la presencia deseada en el futuro cercano. Alcanzar los resultados necesarios en este ámbito, sólo es viable, mediante un sólido conocimiento y el desarrollo de competencias de comunicación y de técnicas de gestión. Si las organizaciones que conducen las capacidades nacionales de información establecen políticas orientadas a potenciar sus recursos humanos y los estilos de trabajo, existirían mejores condiciones para producir un cambio notable en el estilo y comportamiento general de las mismas, en la primera década del próximo milenio.

\section{- la gestión de organizaciones de información}

La gestión está presente en todas nuestras acciones. Se vislumbra que las organizaciones durante el próximo siglo estarán sometidas en forma permanente a cambios discontinuos $\mathrm{y}$ mejoras continuas, con altos niveles de flexibilidad, donde primen las lianzas estratégicas con otras organizaciones, donde se estimule la cooperación, exista una tolerancia ante la ambigüedad y donde el tratamiento a las personas, el liderazgo, la creatividad, la intuición tenga una mayor preponderancia. Elemento importante en estos cambios, será la velocidad y la capacidad de reacción, lo que debe estar muy vinculado al monitoreo del medio y una reinvención permanente de las ventajas.

En esas circunstancias, nuestras unidades de información y bibliotecas deberán ser capaces de asumir esta evolución en el plan de su propia gestión. Sin una gestión exitosa, sería imposible asegurar los niveles de respuesta esperados. El éxito está asociado a un comportamiento favorable y sostenido de determinadas variables asociadas al sistema como son: costes, tiempo y precisión.

También es importante considerar otras variables asociadas a las personas que desarrollan y operan estos sistemas, como: la aptitud (dominio personal, conocimientos, dedicación); la actitud (amor, flexibilidad) y otras conductas y estilos (el trabajo en equipo, el empowerment, la actualización y la investigación) forman parte de esta mezcla de éxito.

Es por estos motivos que resulta fundamental que los profesionales de la información que realizan sus funciones informativas con independencia de la existencia de instituciones de información, y que como tal, tienen un mercado de trabajo mucho más amplio, tracen planes de acción orientados a la gestión del cambio. Definitivamente sin una sólida base teórica y métodos de gestión, y sin el dominio de tecnologías de punta, esto resultaría prácticamente imposible. Sin un perfeccionamiento, sin una actualización continua tampoco sería posible.

\section{BALANCE DE LAS TENDENCIAS Y RE- TOS FUTUROS}

América Latina tenderá en medio de una crisis económica y social a continuar avanzando hacia una sociedad de información. La creación de ese sector de información constituirá el reto mayor para nosotros, los profesionales del nuevo siglo. Nuestra fuerza profesional debe aspirar a ocupar un espacio prioritario dentro de los 
sectores de influencia nacional. Para ello, la elevación de la cultura informacional de los decisores y de la población en general, es una acción estratégica indispensable. ¡No se consume lo que no se conoce!

Nuestros productos y servicios deben transitar por un mayor nivel de agregación de valor. Los profesionales de información debemos tener un mayor impacto en los resultados institucionales y de los usuarios que atendemos. Por tanto generar productos informacionales más complejos es otra de las estrategias a considerar. ¡Necesitamos agregar valor a nuestros productos!

Sobre nuestros hombros recae la responsabilidad de transmitir a las generaciones futuras, con la mayor exactitud y riqueza posible, todo el quehacer científico y social de nuestra Región. ¡Somos defensores de nuestra identidad latinoamericana!

Las nuevas tecnologías de información y comunicación han marcado un nuevo rumbo. ¿Por qué seguir andando por caminos y atajos, si existen grandes autopistas a nuestra disposición? Pero no podemos caminar por autopistas, se hace necesario contar con cierto dominio de las herramientas propias de ese ámbito. Sin sustituir a los especialistas en estos medios, pero sí integrando equipos multidisciplinarios. Somos los gerentes de contenidos. No hay autopistas sin contenidos.

Grupos humanos preparan productos y servicios para otras personas. El conocimiento de técnicas que faciliten la comunicación y el entendimiento es imprescindible. Necesitamos fortalecer y ejercer este rol.

La transparencia, la flexibilidad, la ética, la potenciación de todos constituyen actitudes, la regla y no la excepción. Si aspiramos a un posicionamiento, definitivamente hay que liderar en las organizaciones, en la profesión y dentro de la sociedad. Eso no sería posible sin una sólida base de conocimientos, mejorados continuamente, y sin una sólida cooperación profesional.

Tener éxito equivale a obtener un resultado feliz. Para lograr el éxito es imprescindible desarrollar una gestión eficaz. Sin aplicar métodos y herramientas de gestión resulta improbable lograr resultados óptimos. ¡Seamos eficientes gerentes de información, logrando reducir costos, y elevar beneficios e impacto!

\section{BIBLIOGRAFÍA}

ALTBACH, P. Book publishing. In: UNESCO. World Information Report 1997/98. [S.1.] : Unesco Publishing, 1997. p. 318-327.

AMARAL, S. A . do. Bibliotecas y bibliotecarios especializados en América Latina y el Caribe. Investigación Bibliotecológica.

COOK, M. Access to archival holdings and unique library materials. In: UNESCO. World Information Report 1997/98. [S.1.] : Unesco Publishing, 1997. p. 328-348.

CLERC, P. Economic intelligence. In: UNESCO. World Information Report 1997/98. [S.1.] : Unesco Publishing, 1997. p. 304-317.

DRABENSTOTT, K., BURMAN, C. M. Revisão analítica da biblioteca do futuro. Ciência da Informação, v. 26, n. 2, p. 180-194, 1997. 
DYKSTRA, M. Information highways. In: UNESCO. World Information Report 1997/ 98. [S.1.] : Unesco Publishing, 1997. p. 285303.

FERIA, L., CARVAJAL, M. G., JAUREGUI, M. A . La biblioteca electrónica en Colima México. Ciência da Informação. v. 26, n. 2, p. 165-67, 1997.

GUEVARA, N. Tendencias actuales de la bibliotecología, la archivología y la documentación en el contexto del surgimiento de la ciencia de la información. INFOLAC, v. 8, n. 4, p. 2-8, 1995.

MOORE, N. The information society. In: UNESCO. World Information Report 1997/ 98. [S.1.] : Unesco Publishing, 1997. p. 271284.

MORALES, E. Latin America and the Caribbean. In: UNESCO. World Information Report 1997/98. [S.1.] : Unesco Publishing, 1997. p. $107-123$.

MORALES, E. (comp.). Bibliotecología Latinoamericana: un panorama general. México : CUIB, 1989. 164p.

OPPENHEIM, C. Copyright in the electronic age. In: UNESCO. World Information Report 1997/98. [S.1.] : Unesco Publishing, 1997.p. 349-360.

ROBERTS, K. H. The library in tomorrow's society. A literature review. Paris : UNESCO, 1987. 103p.

RODRÍGUEZ, Victórico. Los servicios de información en el próximo milenio. INFOLAC, v. 10, n. 4, p. 4-12, 1997.

UNESCO. Division des statistiques. Library Statistics in Latin America and the Caribbean. IFLA. Genral Conference, 60th,
Havana, 1994, Booklet 6, p.13-24.

VALENTIM, M. L. P. Assumindo um novo paradigma na biblioteconomia. Informação\&Informação, v. 0, n. 0, p. 2-6, 1995.

VILLALBA, L. Aproximación al estudio de las bibliotecas públicas metropolitanas en América Latina. INFOLAC, v. 9, n. 1, p. 2-8, 1997.

ZAMORA, R. M. F. de. Library resources in Latin America: a general panorama. IFLA Journal, v. 17, n. 1, p. 45-53, 1991.

Este trabalho foi apresentado no InfoChile98, realizado em Santiago, de 02 a 04 de novembro de 1998

e autorizada sua publicação pelo Colegio de Bibliotecarios do Chile 


\section{Gloria Ponjuán Dante}

Centro de Estudios y Desarrollo Profesional en Ciencias de la Información (PROINFO) - IDICT, La Habana, Cuba

\section{Title}

Trends and present situation of the Libraries and Information Units

\section{Abstract}

This article is a synthesis of the general overview of the national, public, school, university libraries and the information units in the 18 Latin American countries. It analyses the Librarians's professional context in these institutions, as well as the elements that are part of the electronic information circle: publishing, copyright, conservation/preservation. It concludes that the information professional has to apply methods and management instruments in order to achieve an effective performance.

\section{Keywords}

Libraries - Latin America; Information Professional - Latin America.

\section{Resumen}

Síntesis del panorama general de las bibliotecas nacionales, públicas, escolares, universitarias y unidades de información en los 18 países participantes de América Latina. Analiza el contexto profesional de los bibliotecarios actuantes en estas instituciones, así como también los elementos integrantes del circuito de la información electrónica: edición, copyright, conservación. Concluye que el profesional de la información debe aplicar métodos e instrumentos de gestión para una actuación eficaz.

\section{Palabras-clave}

Bibliotecas - América Latina; Profesional de la Información - América latina 